\title{
MATRIX LOCALIZATIONS OF $n$-FIRS. I
}

BY

\author{
PETER MALCOLMSON
}

\begin{abstract}
An $n$-fir is an associative ring in which every $n$-generator right ideal is free of unique rank. Matrix localization of a ring involves the adjunction of universal inverses to certain matrices over the ring, so that a new ring results over which the matrices have inverses, but so that the minimum of additional relations is imposed. A full matrix is a square matrix which, when considered as an endomorphism of a free module, cannot be factored through a free module of smaller rank. The main result of this paper is that if the original ring is an $n$-fir with $n>2 k$ and if we form a matrix localization by adjoining universal inverses to all full matrices of size $k$, then the resulting ring is an $(n-2 k)$-fir. This generalizes an announced result of V. N. Gerasimov. There are related results on the structure of the universal skew field of fractions of a semifir.
\end{abstract}

In algebraic geometry, the fact that a polynomial ring embeds in its quotient field is of fundamental importance. Thus there has been interest in embedding a free associative (but noncommutative) algebra in some sort of skew field. While various ways have been found, the method of P. M. Cohn [3] results in the "largest" such skew field, one which specializes to any other. This method, which may be termed "matrix localization", involves the adjunction of inverses to certain matrices over the free algebra (as opposed to the commutative case in which inverses of nonzero elements are adjoined). The method may be applied to any ring, as in the commutative case, resulting in a "localized" ring.

Since the free algebra has the property that its right ideals are free (it is a "fir"), there has been interest in whether a localized ring will have the same sort of property. Cohn and W. Dicks $[4,5]$ have given conditions under which the localization of a fir is again a fir. More recently, V. N. Gerasimov [6] has announced that using matrix localization to invert all nonzero elements of an " $n$-fir" (i.e., a ring for which $n$-generator ideals are free) results in an $(n-2)$-fir. [See Author's Notes below.]

In this article the author intends to generalize the latter result in such a way as to increase the power of the methods available. This is done using earlier work on matrix localizations [7] and a condition on the set of matrices to be inverted which is sufficient to imply that the localized ring is a $p$-fir (for appropriate $p$ ). This is applied to the case of inverting all $k \times k$ full matrices over an $n$-fir to show that the localized ring is an $(n-2 k)$-fir.

Received by the editors June 2, 1982 and, in revised form, August 27, 1982.

1980 Mathematics Subject Classification. Primary 16A06, 16A08.

Key words and phrases. Matrix localization, $n$-fir, universal localization.

(C)1984 American Mathematical Society $0002-9947 / 84 \$ 1.00+\$ .25$ per page 
In the preliminary section we recall the definitions of the relevant terms. The second consists of a description of the main condition on a set of matrices to be inverted, as well as statements of our main results. The third section includes the proofs of these results and the related lemmas. Finally, we give an example showing Gerasimov's result to be the best possible for $n=2$ and suggesting the same for any $n$.

The author wishes to acknowledge the advice and contribution of Jacques Lewin, both in an early letter which suggested some of the techniques used below and in valuable conversations.

Preliminaries. All rings will be associative with unit, and homomorphisms will preserve the unit. Let $R$ be such a ring. An $n$-term relation over $R$ is a row matrix $X$ with $n$ entries and a column matrix $Y$ of the same size, both over $R$, such that $X Y=0$. Such a relation is said to be trivial if for every entry of $X$, either that entry is zero or the corresponding entry of $Y$ is zero. We call an $n$-term relation trivializable if there exists an invertible matrix $Z$ over $R$ such that the $n$-term relation $(X Z)\left(Z^{-1} Y\right)=0$ is trivial.

A ring $R$ such that every $k$-term relation is trivializable for each $k \leqslant n$ is called an $n$-fir. We say $R$ is a semifir if $R$ is an $n$-fir for all $n$. These definitions are from P. M. Cohn's book [3] on such rings and their analogues. In particular we note the result that $R$ is an $n$-fir if and only if every right ideal on $\leqslant n$ generators is a free module on a well-defined number of generators (hence the name fir, an acronym for free ideal rings).

If $X$ is a $k \times n$ matrix and $Y$ an $n \times m$ matrix, both over $R$, and if $X Y=0$ (the zero matrix), we will call this an $n$-term matrix relation. It will be said to be trivial if for every column of $X$, either that column is (all) zero or the corresponding row of $Y$ is zero. It will be called trivializable if there is an invertible matrix $Z$ over $R$ such that $(X Z)\left(Z^{-1} Y\right)=0$ is trivial. It is easy to check by induction that over an $n$-fir every $k$-term $(k \leqslant n)$ matrix relation is trivializable.

Matrix localization involves the formation of universal inverses for certain matrices over $R$ (any ring). Let $\Sigma$ be a collection of square matrices (of various sizes, perhaps) over $R$. A $\Sigma$-inverting $R$-ring is a homomorphism from $R$ to another ring such that the image of each matrix in $\Sigma$ (that is, the result of applying the homomorphism to each entry) is an invertible matrix over the second ring. The universal $\Sigma$-inverting $R$-ring $\lambda: R \rightarrow R_{\Sigma}$ is a $\Sigma$-inverting $R$-ring such that for any $\Sigma$-inverting $R$-ring $R \rightarrow S$ there is a unique ring homomorphism $R_{\Sigma} \rightarrow S$ completing the diagram. This $\lambda$ : $R \rightarrow R_{\Sigma}$ is unique up to appropriate isomorphism. We will refer to $\lambda$ as the universal homomorphism and to $R_{\Sigma}$ as the universal $\Sigma$-inverting ring, or perhaps as the matrix localization.

Cohn [3, p. 255] shows how $\lambda$ and $R_{\Sigma}$ may be constructed by generators and relations. This author has presented a more explicit construction of $R_{\Sigma}$ [7] under the assumption that $\Sigma$ satisfies the following closure condition. We will call $\Sigma$ multiplicative if (1) the $1 \times 1$ identity matrix belongs to $\Sigma$, and if (2) whenever $A$ and $B$ are matrices in $\Sigma$, then the matrix $\left(\begin{array}{ll}A & C \\ 0 & B\end{array}\right)$ belongs to $\Sigma$, where $C$ is any matrix of the appropriate size. 
For any set $\Sigma$ of square matrices, there clearly exists a multiplicative closure of $\Sigma$, the smallest multiplicative set containing $\Sigma$. An element of this closure will have the form

$$
\left(\begin{array}{cccc}
A_{1} & * & * & \\
0 & A_{2} & * & \ddots \\
0 & 0 & A_{3} & \ddots
\end{array}\right),
$$

where each $A_{i}$ is a square block in $\Sigma$ and the *'s denote arbitrary blocks. We will say that this matrix has block upper triangular form with diagonal blocks $A_{1}, A_{2}, \ldots$. Two such matrices will be said to have the same shape if the corresponding diagonal blocks have the same sizes.

We will also call $\Sigma$ factor-closed if whenever $A B$ is in $\Sigma$ for square matrices $A, B$ over $R$, then both $A$ and $B$ are in $\Sigma$.

We will now describe the inner rank function $\rho$ for matrices over $R$. If $X$ is an $n \times m$ matrix over $R$, then $X$ may factor as $X=Y Z$, where $Y$ is an $n \times k$ matrix and $Z$ is $k \times m$, both over $R$. The inner rank $\rho(X)$ is defined to be the minimal $k$ for which this sort of factorization occurs. A square matrix is full if its inner rank is the same as its size.

Inasmuch as an $n \times m$ matrix may be regarded as a map from a free module of rank $m$ to one of rank $n$, we will allow the existence of $n \times m$ matrices with $n=0$ or $m=0$ (or both). Such matrices are said to be null and correspond to zero maps to and from the free module of rank zero. In particular, the inner rank of any zero matrix is zero.

The results. The following is the specialized definition that allows us to carry out our program. A set $\Sigma$ of square matrices over $R$ is said to be $p$-complete if the following trivialization condition holds: for every matrix relation of the form

$$
\left(\begin{array}{lll}
A & X & P \\
S & T & U
\end{array}\right)\left(\begin{array}{cc}
Q & V \\
G & W \\
B & Y
\end{array}\right)=\left(\begin{array}{ll}
0 & 0 \\
0 & 0
\end{array}\right)
$$

where $A$ and $B$ are either null $(0 \times 0)$ or in $\Sigma$, and where $X$ has $\leqslant p$ columns, there exists an invertible matrix $Z$ over $R$ such that

$$
\left(\begin{array}{lll}
A & X & P \\
S & T & U
\end{array}\right) Z=\left(\begin{array}{lll}
A^{\prime} & X^{\prime} & 0 \\
S^{\prime} & T^{\prime} & 0
\end{array}\right) \text { and } Z^{-1}\left(\begin{array}{cc}
Q & V \\
G & W \\
B & Y
\end{array}\right)=\left(\begin{array}{cc}
0 & 0 \\
G^{\prime} & W^{\prime} \\
B^{\prime} & Y^{\prime}
\end{array}\right),
$$

where $A^{\prime}$ and $B^{\prime}$ are either null or in $\Sigma$, and where

$$
\left(\begin{array}{l}
X^{\prime} \\
T^{\prime}
\end{array}\right)\left(\begin{array}{ll}
G^{\prime} & W^{\prime}
\end{array}\right)=\left(\begin{array}{ll}
0 & 0 \\
0 & 0
\end{array}\right)
$$

is a trivial relation. In this definition we intend that the $A, X, P, \ldots$ denote matrices of appropriate sizes over $R$ and that the primed matrices $A^{\prime}, X^{\prime}, \ldots$ have the same sizes as their umprimed counterparts. In view of the similarity to the trivialization for firs, we will refer to this as a "block trivialization". 
Note that $p$-completeness implies $(p-1)$-completeness and that 0 -completeness has meaning ( $X, T, G$ and $W$ are then null). If we take $B$ to be null as well, the definition states that each matrix relation

$$
\left(\begin{array}{l}
A \\
S
\end{array}\right) V=\left(\begin{array}{l}
0 \\
0
\end{array}\right)
$$

is trivializable to

$$
\left(\begin{array}{l}
A^{\prime} \\
S^{\prime}
\end{array}\right) 0=\left(\begin{array}{l}
0 \\
0
\end{array}\right)
$$

Ths implies that $V=0$ and hence that any $A \in \Sigma$ is a non-zero-divisor (on the other side by a symmetrical argument).

We also note that if, in the original definition, $A$ and $B$ are both taken to be null, the result is the statement that every $p$-term matrix relation is trivializable. Hence the existence of a $p$-complete $\Sigma$ necessitates that $R$ be a $p$-fir.

The theorems below give our main results of interest. However, the reader may be interested in some of the lemmas of the following section, particularly Lemmas 5 and 7 .

THEOREM 1. Let $\Sigma$ be a p-complete set of square matrices over $R$. Then $R_{\Sigma}$ is a p-fir, and $R \rightarrow R_{\Sigma}$ is an embedding.

Let us describe some notation for the other results. For each $k$, let $\Phi_{k}$ denote the set of all full $k \times k$ matrices over $R$. Let $R_{k}$ denote the universal $\Phi_{k}$-inverting ring. Let $\Phi_{\infty}$ denote the union of the $\Phi_{k}$ and let $R_{\infty}$ denote the universal $\Phi_{\infty}$-inverting ring.

Now for any ring $S$, we can form the set $\Sigma_{1}$ of $1 \times 1$ nonzero (i.e., full) matrices over $S$. We denote by $S^{(1)}$ the universal $\Sigma_{1}$-inverting ring. Now inductively we can define $S^{(i)}=\left(S^{(i-1)}\right)^{(1)}$ for all $i>1$.

THEOREM 2. Let $R$ be an $n$-fir with $2 k \leqslant n$. Then $\Phi_{k}$ is $(n-2 k)$-complete.

COROLlaRY 1 . Let $R$ be an $n$-fir with $2 k<n$. Then $R_{k}$ and $R^{(k)}$ are $(n-2 k)$-firs.

THEOREM 3. Let $R$ be a semifir. Then there is a commutative diagram of ring homomorphisms as follows:

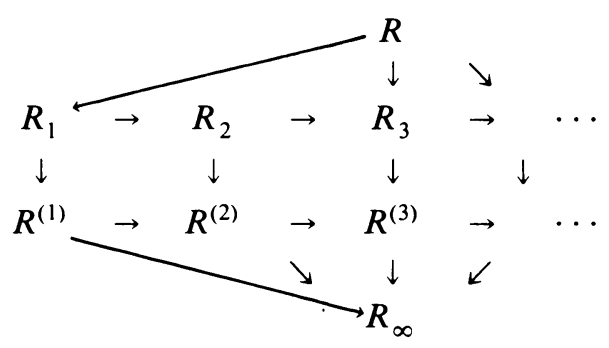

Furthermore, in this diagram all rings are semifirs and all maps are embeddings. Finally, the ring $R_{\infty}$ is a skew field which is the direct limit of either of the two rows. 
The proofs. We use a series of lemmas, each of which has some independent interest.

Lemma 1. Suppose $\Sigma$ is p-complete, and let $\bar{\Sigma}$ denote the multiplicative closure of $\Sigma$. Then $\bar{\Sigma}$ is p-complete, and furthermore the block trivialization required may be accomplished so that $A$ and $A^{\prime}$ (and simultaneously $B$ and $B^{\prime}$ ) have the same shape.

Proof. For the required trivialization, we will proceed by induction on the total number of diagonal blocks in $A$ and in $B$.

If $A$ and $B$ have only one diagonal block each (i.e., are in $\Sigma$ ) or are null, the $p$-completeness of $\Sigma$ provides the necessary trivialization. If (say) $A$ is the identity matrix in

$$
\left(\begin{array}{lll}
I & X & P \\
S & T & U
\end{array}\right)\left(\begin{array}{ll}
Q & V \\
G & W \\
B & Y
\end{array}\right)=\left(\begin{array}{ll}
0 & 0 \\
0 & 0
\end{array}\right),
$$

we may partially trivialize by

$$
\begin{aligned}
\left(\begin{array}{lll}
I & X & P \\
S & T & U
\end{array}\right)\left(\begin{array}{ccc}
I & X & P \\
0 & I & 0 \\
0 & 0 & I
\end{array}\right)^{-1} & =\left(\begin{array}{ccc}
I & 0 & 0 \\
S & T^{\prime} & U^{\prime}
\end{array}\right), \\
\left(\begin{array}{ccc}
I & X & P \\
0 & I & 0 \\
0 & 0 & I
\end{array}\right)\left(\begin{array}{cc}
Q & V \\
G & W \\
B & Y
\end{array}\right) & =\left(\begin{array}{cc}
0 & 0 \\
G & W \\
B & Y
\end{array}\right)
\end{aligned}
$$

and then finish by trivializing

$$
\left(\begin{array}{ll}
T^{\prime} & U^{\prime}
\end{array}\right)\left(\begin{array}{cc}
G & W \\
B & Y
\end{array}\right)=\left(\begin{array}{ll}
0 & 0
\end{array}\right)
$$

(where $A$ is null). We may treat $B$ symmetrically, completing the argument when the minimal number of blocks occur in $A$ and $B$.

Now let us assume $A$ consists of more than one block (again the case for $B$ will be symmetrical). So assume

$$
\left(\begin{array}{cccc}
A_{1} & C & X_{1} & P_{1} \\
0 & A_{2} & X_{2} & P_{2} \\
S_{1} & S_{2} & T & U
\end{array}\right)\left(\begin{array}{cc}
Q_{1} & V_{1} \\
Q_{2} & V_{2} \\
G & W \\
B & Y
\end{array}\right)=\left(\begin{array}{ll}
0 & 0 \\
0 & 0 \\
0 & 0
\end{array}\right),
$$

where $A_{1} \in \bar{\Sigma}, A_{2} \in \Sigma$ (or is an identity matrix) and $B \in \bar{\Sigma}$ (or is null). By induction, the relation

$$
\left(\begin{array}{lll}
A_{2} & X_{2} & P_{2}
\end{array}\right)\left(\begin{array}{cc}
Q_{2} & V_{2} \\
G & W \\
B & Y
\end{array}\right)=(0
$$

may be trivialized to

$$
\left(\begin{array}{lll}
A_{2}^{\prime} & X_{2}^{\prime} & 0
\end{array}\right)\left(\begin{array}{cc}
0 & 0 \\
G^{\prime} & W^{\prime} \\
B^{\prime} & Y^{\prime}
\end{array}\right)=\left(\begin{array}{ll}
0 & 0
\end{array}\right),
$$


where $A_{2}^{\prime} \in \Sigma$ (or is an identity matrix), $B^{\prime} \in \bar{\Sigma}$ having the same shape as $B$, and $X_{2}^{\prime}\left(G^{\prime} W^{\prime}\right)$ trivially zero. If the trivialization is accomplished by the invertible matrix $Z$, then by rearranging rows of $Z$ we may assume $X_{2}^{\prime}$ has the form $\left(\begin{array}{ll}X_{21}^{\prime} & 0\end{array}\right)$ and $\left(G^{\prime} \quad W^{\prime}\right)$ has the form

$$
\left(\begin{array}{cc}
0 & 0 \\
G_{2}^{\prime} & W_{2}^{\prime}
\end{array}\right)
$$

Now using the matrix $\left(\begin{array}{ll}l & 0 \\ 0 & Z\end{array}\right)$, we can partially trivialize the original relation into

$$
\left(\begin{array}{ccccc}
A_{1} & C^{\prime} & X_{11}^{\prime} & X_{12}^{\prime} & P_{1}^{\prime} \\
0 & A_{2}^{\prime} & X_{21}^{\prime} & 0 & 0 \\
S_{1} & S_{2}^{\prime} & T_{1}^{\prime} & T_{2}^{\prime} & U^{\prime}
\end{array}\right)\left(\begin{array}{cc}
Q_{1} & V_{1} \\
0 & 0 \\
0 & 0 \\
G_{2}^{\prime} & W_{2}^{\prime} \\
B^{\prime} & Y^{\prime}
\end{array}\right)=\left(\begin{array}{cc}
0 & 0 \\
0 & 0
\end{array}\right) .
$$

We can use induction again to trivialize

$$
\left(\begin{array}{ccc}
A_{1} & X_{12}^{\prime} & P_{1}^{\prime} \\
S_{1} & T_{2}^{\prime} & U^{\prime}
\end{array}\right)\left(\begin{array}{cc}
Q_{1} & V_{1} \\
G_{2}^{\prime} & W_{2}^{\prime} \\
B^{\prime} & Y^{\prime}
\end{array}\right)=\left(\begin{array}{cc}
0 & 0 \\
0 & 0
\end{array}\right),
$$

obtaining a relation

$$
\left(\begin{array}{ccc}
A_{1}^{\prime} & X_{12}^{\prime \prime} & 0 \\
S_{1}^{\prime} & T_{2}^{\prime \prime} & 0
\end{array}\right)\left(\begin{array}{cc}
0 & 0 \\
G_{2}^{\prime \prime} & W_{2}^{\prime \prime} \\
B^{\prime \prime} & Y^{\prime \prime}
\end{array}\right)=\left(\begin{array}{ll}
0 & 0 \\
0 & 0
\end{array}\right)
$$

where $A_{1}^{\prime} \in \bar{\Sigma}$ has the same shape as $A_{1}, B^{\prime \prime} \in \bar{\Sigma}$ has the same shape as $B^{\prime}$, and

$$
\left(\begin{array}{c}
X_{12}^{\prime \prime} \\
T_{2}^{\prime \prime}
\end{array}\right)\left(\begin{array}{ll}
G_{2}^{\prime \prime} & W_{2}^{\prime \prime}
\end{array}\right)
$$

is trivially zero. The result of this trivialization on the original relation is

$$
\left(\begin{array}{ccccc}
A_{1}^{\prime} & C^{\prime} & X_{11}^{\prime} & X_{12}^{\prime \prime} & 0 \\
0 & A_{2}^{\prime} & X_{21}^{\prime} & 0 & 0 \\
S_{1}^{\prime} & S_{2}^{\prime} & T_{1}^{\prime} & T_{2}^{\prime} & 0
\end{array}\right)\left(\begin{array}{cc}
0 & 0 \\
0 & 0 \\
0 & 0 \\
G_{2}^{\prime \prime} & W_{2}^{\prime \prime} \\
B^{\prime \prime} & Y^{\prime \prime}
\end{array}\right)=\left(\begin{array}{ll}
0 & 0 \\
0 & 0
\end{array}\right),
$$

which is trivialized as desired.

We now wish to use the author's construction of $R_{\Sigma}$ in the following lemma. This construction is based on the result of P. M. Cohen [3] that every element of $R_{\Sigma}$ is of the form $F A^{-1} X$, where $F$ is a row matrix over $R, X$ is a column, and $A$ is an element of the multiplicative closure of $\Sigma$. (Actually, the $F, A, X$ are images in $R_{\Sigma}$ of the appropriate matrices over $R$.) 
Lemma 2. Let $\Sigma$ be 0-complete, $\bar{\Sigma}$ its multiplicative closure and $\lambda: R \rightarrow R_{\Sigma}$ the universal $\Sigma$-inverting homomorphism. Then an element $r$ in $R$ has $\lambda(r)=$ $\lambda(F) \lambda(A)^{-1} \lambda(X)$ (where $A \in \bar{\Sigma}$ and $F, X$ are appropriately sized) in $R_{\Sigma}$ if and only if there exist $P, Q$ of the same shape as $A$, with $P \in \bar{\Sigma}$ and $U, V$, all over $R$, such that

$$
\left(\begin{array}{cc}
A & X \\
F & r
\end{array}\right)=\left(\begin{array}{l}
P \\
U
\end{array}\right)\left(\begin{array}{ll}
Q & V
\end{array}\right)
$$

In particular, $\lambda$ is an embedding.

Proof. The latter factorization implies that $\lambda(A)$ and $\lambda(P)$ are invertible, whence $\lambda(Q)$ is, also. An easy computation in this case shows

$$
\lambda(F) \lambda(A)^{-1} \lambda(X)=\lambda(U) \lambda(V)=\lambda(r) .
$$

Conversely, if $\lambda(F) \lambda(A)^{-1} \lambda(X)=\lambda(r)$, then by the author's construction [7] there exist $L^{\prime}, M^{\prime}, S, T$ in $\bar{\Sigma}$ and $J^{\prime}, W^{\prime}, Y, X$ with

$$
\left(\begin{array}{cccc|c}
A & 0 & 0 & 0 & X \\
0 & 1 & 0 & 0 & -r \\
0 & 0 & L^{\prime} & 0 & 0 \\
0 & 0 & 0 & M^{\prime} & W^{\prime} \\
\hline F & 1 & J^{\prime} & 0 & 0
\end{array}\right)=\left(\frac{S}{Y}\right)(T \mid Z)
$$

Let $S$ have block rows $S_{1}, S_{2}, S_{3}, S_{4}$, compatible with the diagonal blocks $A, 1, L^{\prime}$, $M^{\prime}$, and let the corresponding block rows of the identity matrix be $I_{1}, I_{2}, I_{3}, I_{4}$. Then we have the following factorization:

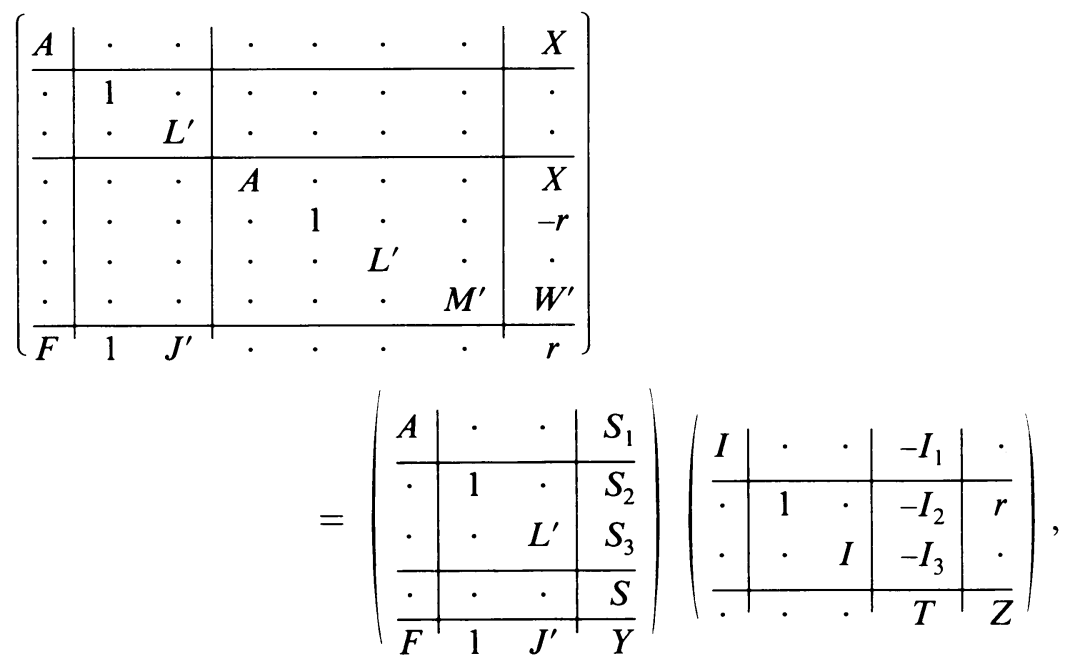

where the dots represent zero blocks. Relabelling the blocks outlined above, we get a factorization

$$
\left(\begin{array}{ccc|c}
A & \cdot & \cdot & X \\
\cdot & L & \cdot & \cdot \\
\cdot & \cdot & M & W \\
\hline F & J & \cdot & r
\end{array}\right)=\left(\begin{array}{ccc}
A_{1} & P_{1} & Q_{1} \\
\cdot & L_{1} & R_{1} \\
\cdot & \cdot & M_{1} \\
\hline F_{1} & G_{1} & H_{1}
\end{array}\right)\left(\begin{array}{ccc|c}
A_{2} & P_{2} & Q_{2} & X_{2} \\
\cdot & L_{2} & R_{2} & Y_{2} \\
\cdot & \cdot & M_{2} & Z_{2}
\end{array}\right),
$$


where $A_{1}, A_{2}, L, M, L_{1}, L_{2}, M_{1}, M_{2}$ are in $\bar{\Sigma}$ and $A_{1}$ and $A_{2}$ have the same shape as A.

Now we use Lemma 1 to trivialize the relation

$$
\left(\begin{array}{ll}
L_{1} & R_{1}
\end{array}\right)\left(\begin{array}{ll}
R_{2} & Y_{2} \\
M_{2} & Z_{2}
\end{array}\right)=(0
$$

to one of the form

$$
\left(\begin{array}{ll}
L_{1}^{\prime} & 0
\end{array}\right)\left(\begin{array}{cc}
0 & 0 \\
M_{2}^{\prime} & Z_{2}^{\prime}
\end{array}\right)
$$

The effect on the original factorization transforms it into

$$
\left(\begin{array}{ccc}
A_{1} & P_{1}^{\prime} & Q_{1}^{\prime} \\
\cdot & L_{1}^{\prime} & \cdot \\
\cdot & N_{1}^{\prime} & M_{1}^{\prime} \\
F_{1} & G_{1}^{\prime} & H_{1}^{\prime}
\end{array}\right)\left(\begin{array}{cccc}
A_{2} & P_{2} & Q_{2} & X_{2} \\
\cdot & L_{2}^{\prime} & \cdot & \cdot \\
\cdot & N_{2}^{\prime} & M_{2}^{\prime} & Z_{2}^{\prime}
\end{array}\right)=\left(\begin{array}{ccc|c}
A & \cdot & \cdot & X \\
\cdot & L & \cdot & \cdot \\
\cdot & \cdot & M & W \\
\hline F & J & \cdot & r
\end{array}\right),
$$

where $L_{1}^{\prime}$ and $M_{2}^{\prime}$ are still in $\bar{\Sigma}$. Now we use the lemma again to trivialize

$$
\left(\begin{array}{ll}
A_{1} & Q_{1}^{\prime} \\
F_{1} & H_{1}^{\prime}
\end{array}\right)\left(\begin{array}{l}
Q_{2} \\
M_{2}^{\prime}
\end{array}\right) \text { into }\left(\begin{array}{ll}
A_{1}^{\prime} & 0 \\
F_{1}^{\prime} & 0
\end{array}\right)\left(\begin{array}{c}
0 \\
M_{2}^{\prime \prime}
\end{array}\right),
$$

and rewrite the original as

$$
\left(\begin{array}{ccc}
A_{1}^{\prime} & P_{1}^{\prime} & \cdot \\
\cdot & L_{1}^{\prime} & \cdot \\
\cdot & N_{1}^{\prime} & M_{1}^{\prime \prime} \\
F_{1}^{\prime} & G_{1}^{\prime} & \cdot
\end{array}\right)\left(\begin{array}{cccc}
A_{2}^{\prime} & P_{2}^{\prime} & \cdot & X_{2}^{\prime} \\
\cdot & L_{2}^{\prime} & \cdot & \cdot \\
K_{2}^{\prime} & N_{2}^{\prime \prime} & M_{2}^{\prime \prime} & Z_{2}^{\prime \prime}
\end{array}\right)=\left(\begin{array}{ccc|c}
A & \cdot & \cdot & X \\
\cdot & L & \cdot & \cdot \\
\cdot & \cdot & M & W \\
\hline F & J & \cdot & r
\end{array}\right) \text {, }
$$

where $A_{1}^{\prime}, M_{2}^{\prime \prime}$ are in $\bar{\Sigma}$ and $A_{1}^{\prime}$ has the same shape as $A_{1}$. From this, it follows that

$$
\left(\begin{array}{ll}
A & X \\
F & r
\end{array}\right)=\left(\begin{array}{l}
A_{1}^{\prime} \\
F_{1}^{\prime}
\end{array}\right)\left(\begin{array}{ll}
A_{2}^{\prime} & X_{2}^{\prime}
\end{array}\right),
$$

as desired. We remark that $A_{2}^{\prime}$ has the same shape as $A$ because $A_{1}^{\prime}$ does, and because the diagonal blocks of $A_{1}^{\prime}$ are non-zero-divisors.

To conclude that $\lambda$ is an embedding, let $\lambda(r)=0$. Then there is a factorization

$$
\left(\begin{array}{ll}
1 & r \\
1 & 0
\end{array}\right)=\left(\begin{array}{l}
p \\
u
\end{array}\right)\left(\begin{array}{ll}
q & v
\end{array}\right)
$$

where $p \in \bar{\Sigma}$ is $1 \times 1$. Since $p q=1$ and $p$ is a non-zero-divisor, $q=p^{-1}=u^{-1}$ and $r=p v=u v=0$.

If $\Sigma$ is assumed to be factor-closed in Lemma 2, then we can easily see that $Q$ is an $\bar{\Sigma}$, as well. Even a weaker condition will do; we can assume that if $A=B C$ with $A, B, C$, square and $A, B \in \Sigma$, then $C \in \Sigma$. We get a similar condition from our completeness assumption.

Lemma 3. Let $A=B C$, where $A, B$, and $C$ are square matrices and $A, B \in \Sigma$. If $\Sigma$ is 0 -complete, then there is an invertible matrix $E$ with $E C \in \Sigma$. 
Proof. The matrix relation

$$
\left(\begin{array}{ll}
B & I
\end{array}\right)\left(\begin{array}{c}
-C \\
A
\end{array}\right)=0
$$

is trivializable, say by the invertible matrix

$$
Z=\left(\begin{array}{ll}
Z_{11} & Z_{12} \\
Z_{21} & Z_{22}
\end{array}\right) .
$$

Then $\left(\begin{array}{ll}B & I\end{array}\right) Z=\left(\begin{array}{ll}B^{\prime} & 0\end{array}\right)$ with $B^{\prime} \in \Sigma$. So

$$
\left(\begin{array}{ll}
B & I \\
I & 0
\end{array}\right) Z=\left(\begin{array}{ll}
B^{\prime} & 0 \\
Z_{11} & Z_{12}
\end{array}\right)
$$

is invertible. Since $B^{\prime} \in \Sigma$ is a non-zero-divisor, $B^{\prime}$ is invertible and so is $Z_{12}$. We now use

$$
Z^{-1}\left(\begin{array}{c}
-C \\
A
\end{array}\right)=\left(\begin{array}{c}
0 \\
A^{\prime}
\end{array}\right)
$$

with $A^{\prime} \in \Sigma$, or

$$
\left(\begin{array}{c}
-C \\
A
\end{array}\right)=Z\left(\begin{array}{c}
0 \\
A^{\prime}
\end{array}\right)
$$

That shows $-C=Z_{12} A^{\prime}$, finishing the proof.

Lemma 4. Over any ring, suppose $\left(\begin{array}{ll}A & X\end{array}\right) Z=\left(\begin{array}{ll}B & Y\end{array}\right)$, where $A, B$ and $Z$ are invertible square matrices and $X, Y$ are compatibly sized blocks. Then the matrix

$$
\left(\begin{array}{ll}
0 & I
\end{array}\right) Z\left(\begin{array}{c}
-B^{-1} Y \\
I
\end{array}\right)
$$

is invertible with inverse

$$
\left(\begin{array}{ll}
0 & I
\end{array}\right) Z^{-1}\left(\begin{array}{c}
-A^{-1} X \\
I
\end{array}\right)
$$

(Symmetrically, if

$$
Z\left(\begin{array}{l}
X \\
A
\end{array}\right)=\left(\begin{array}{c}
Y \\
B
\end{array}\right)
$$

then

$$
\left(\begin{array}{ll}
I & -Y B^{-1}
\end{array}\right) Z\left(\begin{array}{l}
I \\
0
\end{array}\right)
$$

has inverse

$$
\left(\begin{array}{ll}
I & -X A^{-1}
\end{array}\right) Z^{-1}\left(\begin{array}{l}
I \\
0
\end{array}\right)
$$

Proof. If we put

$$
Z=\left(\begin{array}{ll}
Z_{11} & Z_{12} \\
Z_{21} & Z_{22}
\end{array}\right),
$$

then let $D=Z_{21} B^{-1}$ and $C=Z_{22}-D Y$. From this we get

$$
\left(\begin{array}{cc}
A & X \\
0 & I
\end{array}\right) Z=\left(\begin{array}{cc}
I & 0 \\
D & C
\end{array}\right)\left(\begin{array}{cc}
B & Y \\
0 & I
\end{array}\right)
$$


whence $C$ is invertible and

$$
C=\left(\begin{array}{ll}
0 & I
\end{array}\right) Z\left(\begin{array}{c}
-B^{-1} Y \\
I
\end{array}\right)
$$

Proof of TheOrem 1. The homomorphism $\lambda: R \rightarrow R_{\Sigma}$ is an embedding by Lemma 2; we will suppress the notation for $\lambda$ in what follows. Suppose we have an $m$-term relation in $R_{\Sigma}$, with $m \leqslant p$. Write it as $\Sigma\left(f_{i} A_{i}^{-1} x_{i}\right)\left(g_{i} B_{i}^{-1} y_{i}\right)=0$ with $A_{i}$, $B_{i} \in \bar{\Sigma}$, the multiplicative closure of $\Sigma$. Let $A$ be the matrix with diagonal blocks $A_{i}$, $F$ the row consisting of the rows $f_{i}$ arranged consecutively, $X$ the matrix whose columns are the $x_{i}$ 's. Similarly, we can form $G$ (whose rows are the $g_{i}$ 's), $B$ (with diagonal blocks $B_{i}$ ), and $Y$ (a column of the $y_{i}$ ). Then we have the $m$-term relation $\left(F A^{-1} X\right)\left(G B^{-1} Y\right)=0$, with $A, B \in \bar{\Sigma}$, and we can easily compute that

$$
\left(\begin{array}{ll}
F & 0
\end{array}\right)\left(\begin{array}{cc}
A & -X G \\
0 & B
\end{array}\right)^{-1}\left(\begin{array}{l}
0 \\
Y
\end{array}\right)=0 \quad \text { in } R_{\Sigma}
$$

According to Lemma 2 there is a factorization

$$
\left(\begin{array}{ccc}
A & -X G & 0 \\
0 & B & Y \\
F & 0 & 0
\end{array}\right)=\left(\begin{array}{cc}
A_{1} & P \\
0 & B_{1} \\
T & U
\end{array}\right)\left(\begin{array}{ccc}
A_{2} & Q & V \\
0 & B_{2} & W
\end{array}\right)
$$

over $R$, where $A_{1}, B_{1} \in \bar{\Sigma}$. By Lemma $1, \bar{\Sigma}$ is $p$-complete, so by Lemma 3 there is an invertible $E$ with $E B_{2} \in \bar{\Sigma}$. In (*) we can replace $B_{2}$ by $E B_{2}, W$ by $E W, P$ by $P E^{-1}$, and so forth, or, equivalently, in (*) we may assume $A_{1}, B_{2} \in \bar{\Sigma}$.

Now we have

$$
\left(\begin{array}{ccc}
A_{1} & X & P \\
T & 0 & U
\end{array}\right)\left(\begin{array}{cc}
Q & V \\
G & 0 \\
B_{2} & W
\end{array}\right)=\left(\begin{array}{cc}
0 & 0 \\
0 & 0
\end{array}\right)
$$

By $p$-completeness of $\bar{\Sigma}$, there exists an invertible $Z$ with

$$
\left(\begin{array}{ccc}
A_{1} & X & P \\
T & 0 & U
\end{array}\right) Z=\left(\begin{array}{ccc}
A_{1}^{\prime} & X^{\prime} & 0 \\
T^{\prime} & U^{\prime} & 0
\end{array}\right), \quad Z^{-1}\left(\begin{array}{cc}
Q & V \\
G & 0 \\
B_{2} & W
\end{array}\right)=\left(\begin{array}{cc}
0 & 0 \\
G^{\prime} & V^{\prime} \\
B_{2}^{\prime} & W^{\prime}
\end{array}\right)
$$

where $A_{1}^{\prime}, B_{2}^{\prime} \in \bar{\Sigma}$ and

$$
\left(\begin{array}{l}
X^{\prime} \\
U^{\prime}
\end{array}\right)\left(\begin{array}{ll}
G^{\prime} & V^{\prime}
\end{array}\right)=0
$$

is trivial. Using Lemma 4 over $R_{\Sigma}$,

$$
\left(\begin{array}{ccc}
0 & I & 0 \\
0 & 0 & I
\end{array}\right) Z\left(\begin{array}{cc}
-A_{1}^{\prime-1} X^{\prime} & 0 \\
I & 0 \\
0 & I
\end{array}\right)
$$

has inverse

$$
\left(\begin{array}{ccc}
0 & I & 0 \\
0 & 0 & I
\end{array}\right) Z^{-1}\left(\begin{array}{cc}
-A_{1}^{-1} X & -A_{1}^{-1} P \\
I & 0 \\
0 & I
\end{array}\right)=C
$$


say. Computing shows that

$$
C\left(\begin{array}{c}
G \\
B_{2}
\end{array}\right)=\left(\begin{array}{c}
G^{\prime} \\
B_{2}^{\prime}
\end{array}\right)
$$

so Lemma 4 shows that

$$
\left(\begin{array}{ll}
I & -G^{\prime} B_{2}^{\prime-1}
\end{array}\right) C\left(\begin{array}{l}
I \\
0
\end{array}\right)=\left(\begin{array}{lll}
0 & I & G^{\prime} B_{2}^{\prime-1}
\end{array}\right) Z^{-1}\left(\begin{array}{c}
-A_{1}^{-1} X \\
I \\
0
\end{array}\right)
$$

has inverse (over $R_{\Sigma}$ )

$$
\left(\begin{array}{lll}
0 & I & -G B_{2}^{-1}
\end{array}\right) Z\left(\begin{array}{c}
-A_{1}^{\prime-1} X^{\prime} \\
I \\
0
\end{array}\right)
$$

Now the $m$-term relation $\left(F A^{-1} X\right)\left(G B^{-1} Y\right)=0$ can be adjusted by the above matrices into

$$
F A^{-1} X\left(\begin{array}{lll}
0 & I & -G B_{2}^{-1}
\end{array}\right) Z\left(\begin{array}{c}
-A_{1}^{\prime-1} X^{\prime} \\
I \\
0
\end{array}\right)\left(\begin{array}{lll}
0 & I & -G^{\prime} B_{2}^{\prime-1}
\end{array}\right) Z^{-1}\left(\begin{array}{c}
-A_{1}^{-1} X \\
I \\
0
\end{array}\right) G B^{-1} Y
$$

which we claim is trivially zero. To see this, first note that all of $A_{1}, A_{2}, B_{1}, B_{2}, A_{1}^{\prime}$, $B_{2}^{\prime}$ are invertible over $R_{\Sigma}$. Now compute

$$
\begin{aligned}
F A^{-1} X\left(\begin{array}{lll}
0 & I & -G B_{2}^{-1}
\end{array}\right) & =F A^{-1}\left(\begin{array}{lll}
A_{1} & X & P
\end{array}\right)+F A^{-1}\left(\begin{array}{llll}
-A_{1} & 0 & A_{1} Q B_{2}^{-1}
\end{array}\right) \\
& =F A^{-1}\left(\begin{array}{llll}
A_{1} & X & P
\end{array}\right)+\left(\begin{array}{llll}
-T & 0 & -U
\end{array}\right) .
\end{aligned}
$$

Then

$$
F A^{-1} X\left(\begin{array}{lll}
0 & I & -G B_{2}^{-1}
\end{array}\right) Z\left(\begin{array}{c}
-A_{1}^{\prime-1} X^{\prime} \\
I \\
0
\end{array}\right)=\left(\begin{array}{ll}
T^{\prime} A_{1}^{\prime-1} & -I
\end{array}\right)\left(\begin{array}{c}
X^{\prime} \\
U^{\prime}
\end{array}\right)
$$

by more computation. Similarly,

$$
\left(\begin{array}{lll}
0 & I & -G^{\prime} B_{2}^{\prime-1}
\end{array}\right) Z^{-1}\left(\begin{array}{c}
-A_{1}^{-1} X \\
I \\
0
\end{array}\right) G B^{-1} Y=\left(\begin{array}{ll}
G^{\prime} & V^{\prime}
\end{array}\right)\left(\begin{array}{c}
B_{2}^{\prime-1} W^{\prime} \\
-I
\end{array}\right) .
$$

But

$$
\left(\begin{array}{l}
X^{\prime} \\
U^{\prime}
\end{array}\right)\left(\begin{array}{ll}
G^{\prime} & V^{\prime}
\end{array}\right)
$$

is already trivially zero, completing the claim. Thus $R_{\Sigma}$ is a $p$-fir.

We now proceed to results on the inner rank function over an $n$-fir which allow us to prove Theorem 2. These are similar to the algebraic rank functions of [8].

LEMMA 5. Let $\rho$ denote inner rank over an $n$-fir. Then $\rho$ satisfies:

(1) $\rho(0)=0$;

(2) $\rho(1)=1$;

(3) $\rho(a b) \leqslant \operatorname{MIN}(\rho(a), \rho(b))$ for any matrices $a, b$; 
(4) $\rho\left(\begin{array}{ll}a & 0 \\ 0 & b\end{array}\right) \leqslant \rho(a)+\rho(b)$ for any matrices $a, b$;

(5) if $\rho\left(\begin{array}{ll}a & c \\ 0 & b\end{array}\right) \leqslant n$, then

$$
\rho\left(\begin{array}{ll}
a & c \\
0 & b
\end{array}\right) \geqslant \rho(a)+\rho(b)
$$

for any matrices $a, b, c$.

Proof. All are straightforward (indeed, true over any nonzero ring) except (5). Let

$$
\rho\left(\begin{array}{ll}
a & c \\
0 & b
\end{array}\right)=k \leqslant n
$$

so that there is an appropriate factorization

$$
\left(\begin{array}{ll}
a & c \\
0 & b
\end{array}\right)=\left(\begin{array}{l}
x \\
y
\end{array}\right)\left(\begin{array}{ll}
z & w
\end{array}\right)
$$

Then $y z=0$ is a $k$-term matrix relation. We can trivialize this and apply the appropriate invertible matrix to the whole factorization (perhaps rearranging rows/columns if necessary) to obtain a new factorization

$$
\left(\begin{array}{ll}
a & c \\
0 & b
\end{array}\right)=\left(\begin{array}{cc}
x_{1}^{\prime} & x_{2}^{\prime} \\
0 & y_{2}^{\prime}
\end{array}\right)\left(\begin{array}{cc}
z_{1}^{\prime} & w_{1}^{\prime} \\
0 & w_{2}^{\prime}
\end{array}\right)
$$

Denote by $k_{1}$ the number of columns in $x_{1}^{\prime}$, and by $k_{2}$ the number of columns in $y_{2}^{\prime}$. Then $a=x_{1}^{\prime} z_{1}^{\prime}$ and $b=y_{2}^{\prime} w_{2}^{\prime}$ imply

$$
\rho\left(\begin{array}{ll}
a & c \\
0 & b
\end{array}\right)=k=k_{1}+k_{2} \geqslant \rho(a)+\rho(b) .
$$

Note that as a result of (3), elementary row and column operations may be performed on a matrix without changing its inner rank.

Lemma 6. Let $R$ be an $n$-fir, $\rho$ its inner rank. Let $A$ be a matrix over $R$ and $a_{1}, a_{2}$ columns over $R$ with the same number of rows as $A$. If $\rho\left(\begin{array}{ll}A & a_{1}\end{array}\right)+\rho\left(\begin{array}{ll}A & a_{2}\end{array}\right) \leqslant n$, then

$$
\rho\left(\begin{array}{lll}
A & a_{1} & a_{2}
\end{array}\right) \leqslant \rho\left(\begin{array}{ll}
A & a_{1}
\end{array}\right)+\rho\left(\begin{array}{ll}
A & a_{2}
\end{array}\right)-\rho(A) .
$$

Proof. Using (1)-(5) of Lemma 5,

$$
\begin{aligned}
\rho\left(\begin{array}{ll}
A & a_{1}
\end{array}\right)+\rho\left(\begin{array}{ll}
A & a_{2}
\end{array}\right) \geqslant \rho\left(\begin{array}{cccc}
A & a_{2} & 0 & 0 \\
0 & 0 & A & a_{1}
\end{array}\right)=\rho\left(\begin{array}{cccc}
A & 0 & 0 & a_{2} \\
0 & A & a_{1} & 0
\end{array}\right) \\
=\rho\left(\begin{array}{lllll}
A & 0 & 0 & a_{2} \\
A & A & a_{1} & a_{2}
\end{array}\right)=\rho\left(\begin{array}{cccc}
A & 0 & 0 & a_{2} \\
0 & A & a_{1} & a_{2}
\end{array}\right) \geqslant \rho(A)+\rho\left(\begin{array}{lll}
a_{1} & a_{2}
\end{array}\right) .
\end{aligned}
$$

Note that a similar statement holds for rows. We use these to prove a relationship between inner rank and full submatrices.

LEMMA 7. Let $R$ be an $n$-fir, $\rho$ its inner rank. If $A$ is a matrix over $R$ with largest full submatrix of size $k \leqslant n / 2$, then $\rho(A)=k$. 
Proof. In any case $\rho(A) \geqslant k$. Let us assume $A$ is a minimal size counterexample to the lemma. Clearly $A$ is not $1 \times 1$. Let $A_{0}$ be a full submatrix of $A$ of maximal size $k$. Rearrange $A$ as

$$
A=\left(\begin{array}{ll}
A_{0} & A_{1} \\
A_{2} & A_{3}
\end{array}\right)
$$

with $\rho(A)>k$ by assumption. Clearly neither $A_{1}$ nor $A_{2}$ is null.

If $A_{1}$ has more than one column, then by the minimality of $A$ the matrix obtained from $A$ by eliminating one or two columns of $A_{1}$ has inner rank $k$. This would contradict Lemma 6. Similarly, $A_{2}$ cannot have more than one row. Since $A$ cannot be full by the choice of $A_{0}, \rho(A)<k+1$, which contradicts the original assumption.

Proof of Theorem 2. Suppose

$$
\left(\begin{array}{lll}
A & X & P \\
S & T & U
\end{array}\right)\left(\begin{array}{cc}
Q & V \\
G & W \\
B & Y
\end{array}\right)=\left(\begin{array}{ll}
0 & 0 \\
0 & 0
\end{array}\right)
$$

where $A, B \in \Phi_{k}$ (or null) and $X$ has $m \leqslant n-2 k$ columns. The entire matrix relation is $\leqslant n$-term, so it is trivializable as desired, except for the requirement that $A^{\prime}, B^{\prime}$ in the trivialized relation

$$
\left(\begin{array}{ccc}
A^{\prime} & X^{\prime} & 0 \\
S^{\prime} & T^{\prime} & 0
\end{array}\right)\left(\begin{array}{cc}
0 & 0 \\
G^{\prime} & W^{\prime} \\
B^{\prime} & Y^{\prime}
\end{array}\right)=\left(\begin{array}{ll}
0 & 0 \\
0 & 0
\end{array}\right)
$$

can be taken to be full. The inner rank of $\left(\begin{array}{lll}A & X & P\end{array}\right)$ is $k$, since $A$ is full. This is the same as the inner rank of $\left(\begin{array}{lll}A^{\prime} & X^{\prime} & 0\end{array}\right)$ and $2 k \leqslant n$, so there is a full submatrix of ( $A^{\prime} \quad X^{\prime} \quad 0$ ) of size $k$ by Lemma 7 . We may assume this submatrix is $A^{\prime}$ by rearranging columns. The nonzero rows of

$$
\left(\begin{array}{c}
0 \\
G^{\prime} \\
B^{\prime}
\end{array}\right)
$$

may be simultaneously rearranged to force $B^{\prime}$ to be full. (We remark that if $A$ or $B$ is null the situation is easier.)

The proof of Corollary 1 is clear, using induction for $R^{(k)}$. We prove two more lemmas for Theorem 3.

LeMma 8. Let $R$ be an $n$-fir, $A$ a full matrix of size $k \leqslant n / 2+2$. Then there is a full (square) submatrix of $A$ of size $k-1$.

Proof. Let the largest full proper submatrix of $A$ have size $l \geqslant 0$. Assume $l \leqslant k-2$; then $l \leqslant n / 2$. By Lemma 7 every proper submatrix of $A$ has inner rank $\leqslant l$. Since $l \leqslant k-2$, there is a $k \times(k-2)$ submatrix of $A$ with inner rank $l$. Now an application of Lemma 6 shows that the inner rank of $a$ is $\leqslant l$, a contradiction.

The last lemma is a slight generalization of a result of Cohn and W. Dicks [5, Lemma 4]. An honest embedding is a ring embedding such that full matrices have full images. 
To state the lemma in greater generality, recall that a stably-free module is a module $M$ such that the direct sum of $M$ with some finitely generated free module is isomorphic to some finitely generated free module.

Lemma 9. Let $R \rightarrow S$ be an honest embedding and let $\Sigma$ be a set of matrices over $R$ whose images are invertible over $S$. If the universal $\Sigma$-inverting ring $R_{\Sigma}$ satisfies the condition that every stably-free module is free on a well-defined number of generators, then the induced map $R_{\Sigma} \rightarrow S$ is an honest embedding.

Proof. We will suppress the notation for the homomorphisms involved, indicating explicitly in which ring relations hold. As in the proof of Theorem 2, any matrix over $R_{\Sigma}$ may be written in the form $F A^{-1} X$, where $A$ is in the multiplicative closure of $\Sigma$ and $F, X$ are over $R$. Assume now that the image of $F A^{-1} X$ is a (square) nonfull matrix over $S$. Then the matrix

$$
\left(\begin{array}{cc}
A & X \\
F & 0
\end{array}\right)=\left(\begin{array}{cc}
A & 0 \\
F & I
\end{array}\right)\left(\begin{array}{cc}
I & 0 \\
0 & F A^{-1} X
\end{array}\right)\left(\begin{array}{cc}
I & A^{-1} X \\
0 & -I
\end{array}\right)
$$

is nonfull over $S$. By the honesty assumption, the matrix is nonfull over $R$, say

$$
\left(\begin{array}{cc}
A & X \\
F & 0
\end{array}\right)\left(\begin{array}{l}
P \\
U
\end{array}\right)=\left(\begin{array}{ll}
Q & V
\end{array}\right)
$$

Now over $R_{\Sigma}$ we can see that

$$
\left(\begin{array}{cc}
I & 0 \\
0 & F A^{-1} X
\end{array}\right)=\left(\begin{array}{c}
A^{-1} P \\
U-F A^{-1} P
\end{array}\right)\left(\begin{array}{ll}
Q & Q A^{-1} X-V
\end{array}\right) .
$$

The assumption on $R_{\Sigma}$ implies that the matrices $A^{-1} P$ and $Q$ can be "filled out" to form a pair of inverse matrices, say by

$$
\left(\begin{array}{c}
A^{-1} P \\
K
\end{array}\right)^{-1}=(Q
$$

(see [5]). Then by checking sizes we can verify that

$$
\begin{aligned}
\left(\begin{array}{cc}
I & 0 \\
0 & F A^{-1} X
\end{array}\right) & =\left(\begin{array}{c}
A^{-1} P \\
U-F A^{-1} P
\end{array}\right)\left(\begin{array}{cc}
Q & L
\end{array}\right)\left(\begin{array}{c}
A^{-1} P \\
K
\end{array}\right)\left(\begin{array}{ll}
Q & Q A^{-1} X-V
\end{array}\right) \\
& =\left(\begin{array}{cc}
I & 0 \\
0 & U L
\end{array}\right)\left(\begin{array}{cc}
I & 0 \\
0 & -K V
\end{array}\right)
\end{aligned}
$$

shows $F A^{-1} X$ to be nonfull over $R_{\Sigma}$. This checks the honesty of the homomorphisms; for the embedding condition we can take $F A^{-1} X$ to be $1 \times 1$ and follow the above proof with $K, L$ null.

Proof of Theorem 3. P. M. Cohn has shown [3] (or see [9]) that $R_{\infty}$ is a skew field (and thus a semifir) and that the universal map $R \rightarrow R_{\infty}$ is an honest embedding. Since all the $R_{k}$ are semifirs by Corollary 1, they satisfy the condition of Lemma 9, so we get embeddings $R \rightarrow R_{k} \rightarrow R_{\infty}$. Similarly, but by induction this time, the $R^{(k)}$ are semifirs and we get (honest) embeddings $R \rightarrow R^{(k)} \rightarrow R_{\infty}$.

If $A \in \Phi_{k}$, then Lemma 5(5) shows

$$
\left(\begin{array}{cc}
A & 0 \\
0 & 1
\end{array}\right) \in \Phi_{k+1}
$$


Computation shows

$$
\left(\begin{array}{ll}
A & 0 \\
0 & 1
\end{array}\right)^{-1}=\left(\begin{array}{cc}
A^{-1} & 0 \\
0 & 1
\end{array}\right)
$$

so $A$ is invertible over $R_{k+1}$. Then universality gives the map $R_{k} \rightarrow R_{k+1}$. We remark that (as will continue throughout the proof) commutativity of diagrams is established by the uniqueness of universally-defined maps.

Suppose by induction that we have defined the map $R_{k} \rightarrow R^{(k)}$. If $A \in \Phi_{k+1}$, then by Lemma 8 and rearrangement we may assume

$$
A=\left(\begin{array}{ll}
B & C \\
D & E
\end{array}\right)
$$

where $B$ is full of size $k$. For convenience use the same notation for the image of $A$ over $R^{(k)}$. Then

$$
A=\left(\begin{array}{cc}
B & C \\
D & E
\end{array}\right)=\left(\begin{array}{cc}
B & 0 \\
D & 1
\end{array}\right)\left(\begin{array}{cc}
I & B^{-1} C \\
0 & E-D B^{-1} C
\end{array}\right),
$$

where $B^{-1}$ exists over $R^{(k)}$ by the induction hypothesis.

If $E-D B^{-1} C=0$ in $R^{(k)}$, then $A$ is not full over $R^{(k)}$, which is impossible since $A$ is invertible over the skew field $R_{\infty}$ (or by honesty). So $E-D B^{-1} C \neq 0$, and the image of $A$ over $R^{(k+1)}$ is invertible. Universality now provides the map $R_{k+1} \rightarrow$ $R^{(k+1)}$.

All maps can now be seen to be embeddings by the commutativity of the diagram (which follows as before). That $R_{\infty}$ is the direct limit of the upper row is clear; but then the lower row is "squeezed" between the upper row and its limit, proving that the limit for both rows is the same.

REMARKS ON COUNTEREXAMPLES. In this section we give some justification that the result of Corollary 1 is best possible. In particular we will give examples of a 2 -fir $R$ such that $R_{1}$ is not a l-fir (i.e., not a domain).

Let $R$ be the algebra over a field $k$ generated by 18 (noncommuting) variables which comprise the entries of two $3 \times 3$ matrices $C, D$ (over $R$ by definition) and satisfying the 9 relations given by the equation $C D=0$. If we let $L$ be the $k$-algebra generated by noncommuting variables $c, d$ under the relation $c d=0$, then $R$ is (isomorphic to) the "coefficient ring" $W_{3}(L)$ as defined by G. Bergman [1]. This ring has the universal property that homomorphisms from $R$ to any ring $M$ are in 1-1 correspondence with homomorphisms from $L$ to $m_{3}(M)$, the ring of $3 \times 3$ matrices over $M$. By results of Bergman or by the original work of Cohn [2], it may be seen that $R$ is a 2 -fir (though not a 3-fir).

By Theorem 2 the set $\Phi_{1}$ of nonzero (full) $1 \times 1$ matrices over $R$ is 0 -complete. Let us write the first two rows of $C$ as

$$
\left(\begin{array}{lll}
a & x & p \\
s & t & u
\end{array}\right)
$$

and the first two columns of $D$ as

$$
\left(\begin{array}{ll}
q & v \\
g & w \\
b & y
\end{array}\right)
$$


By Lemma 2 the map from $R$ to the universal $\Phi_{1}$-inverting ring $R_{1}$ is an embedding, so we will use the same notation for the images. Then in $R_{1}$,

$$
\begin{aligned}
\left(t-s a^{-1} x\right)\left(w-g b^{-1} y\right) & =t w-s a^{-1} x w-t g b^{-1} y+s a^{-1} x g b^{-1} y \\
= & t w+s a^{-1}(a v+p y)+(s q+u b) b^{-1} y-s a^{-1}(a q+p b) b^{-1} y \\
= & t w+s v+u y=0 .
\end{aligned}
$$

We may see $t-s a^{-1} x$ and $w-g b^{-1} y$ are not zero by using the universal property of $R$ and of $R_{1}$, but it is easier to use Lemma 2. If $t-s a^{-1} x=0$ in $R_{1}$, then we get $\left(\begin{array}{cc}a x \\ s\end{array}\right)$ not full over $R$. This is impossible, since we can map $C$ to $I$ and $D$ to 0 over $k$, obtaining a map from $R$ to $k$ for which the image of $\left(\begin{array}{ll}a & x \\ s & t\end{array}\right)$ is full. The argument that $w-g b^{-1} y \neq 0$ is similar (though using a different homomorphism), so $R_{1}$ is not a domain.

This method easily extends to show that over a $2 k$-fir the universal $\Phi_{k}$-inverting ring need not be a domain. When the method is applied to the appropriate universal $(2 k+p)$-fir and we get a $p$-fir by localizing, we also get a $(p+1)$-term relation which appears to be nontrivializable. However, the proof of this claim seems to be beyond the level of the present techniques.

AuthoR's Notes (added in proof). The first description of Gerasimov's results (appearing in [6]) that $I$ received was in the form stated in the introduction to this paper. It now appears that his results are quite as general as Theorems 1 and 2 above. Thus the new results of this paper include only Theorem 3 and the discussion of counterexamples (aside from various lemmas).

The author also notes that the techniques required to complete the discussion of the above counterexample(s) will appear in another paper (to follow in this journal).

\section{REFERENCES}

1. G. M. Bergman, Coproducts and some universal ring constructions, Trans. Amer. Math. Soc. 200 (1974), 33-88.

2. P. M. Cohn, Dependence in rings. II: The dependence number, Trans. Amer. Math. Soc. 135 (1969), 267-279.

3. __ Free rings and their relations, Academic Press, New York, 1971.

4. L_ Localization in semifirs, Bull. London Math. Soc. 6 (1974), 13-20.

5. P. M. Cohn and W. Dicks, Localization in semifirs. II, J. London Math. Soc. (2) 13 (1976), 411-418

6. V. N. Gerasimov, Localizations of associative rings, Siberian Math J. 23 (1982), 36-55. (Russian)

7. P. Malcolmson, Construction of universal matrix localizations, Lecture Notes in Math., vol. 951, Springer-Verlag, Berlin, 1982.

8. __ Determining homomorphisms to skew fields, J. Algebra 64 (1980), 399-413.

9.

Department of Mathematics, Wayne State University, Detroit, Michigan 48202 